\title{
$X(3872)$ in Heavy Quark Limit of QCD: Its Partners and Isospin Structure
}

\author{
A. Ozpineci $i^{1, a}$ \\ ${ }^{1}$ Department of Physics, Middle East Technical University, Ankara, Turkey
}

\begin{abstract}
Although it has been more than ten years since the discovery of the $X(3872)$ meson, its properties still contain puzzles. In this work, the results obtained using a correlation function approach on the degenerate partners of the $X(3872)$ will be presented. The isospin structure is also discussed in the same framework. Finally, the $X(3872) \rightarrow$ $D^{0} \bar{D}^{0} \pi$ decay is proposed to study the isospin structure of the $X(3872)$ meson.
\end{abstract}

\section{Introduction}

The $X(3872)$ meson was first discovered by BELLE [1] more than ten years ago. Although it has been more than ten years since its discovery, its quantum numbers could only be determined to be $J^{P C}=1^{++}$only recently [2]. One of the interesting puzzles about the $X(3872)$ mesons is its decay into final states containing $J / \Psi$ and pions. $X(3872)$ has been observed to decay into $J / \Psi \pi \pi$ and $J / \Psi \pi \pi \pi$ through $\rho$ and $\omega$ mesons, respectively, with almost equal branching ratios [3]:

$$
\frac{B(X(3872) \rightarrow J / \psi \rho)}{B(X(3872) \rightarrow J / \psi \omega)} \simeq 1
$$

Taking into account the phase space differences between the $\rho$ and $\omega$ mesons, this branching ratio amounts to the following ratio for the amplitudes:

$$
\frac{A(X(3872) \rightarrow J / \psi \rho)}{A(X(3872) \rightarrow J / \psi \omega)} \simeq 0.2
$$

This implies that there is a large isospin violation in the $X(3872)$ meson system.

One popular description of the $X(3872)$ is as a molecule consisting of a $D$ and a $\bar{D}^{*}$ meson in a $J^{P C}=1^{++}$configuration. In this picture, isospin violation naturally arises due to the mass difference between the charged and neutral $D \bar{D}^{*}$ components. In this picture one assumes that the interactions are isospin conserving, but this mass difference creates isospin violation in the kinetic term for the mesons. In $[4,5]$ it was also shown that in the heavy quark limit, there should be three other mesons that have the same binding energy as the $X(3872)$ meson.

In the heavy quark limit, the spin of the heavy quark decouples from the theory, i.e. the physical properties can not depend on the spin. If one considers a system of two heavy quarks, their total spin is conserved and can be either $S_{h}=0$ or $S_{h}=1$. If there are additional two quarks in the system, they

\footnotetext{
ae-mail: ozpineci@metu.edu.tr
} 
Table 1. Spectrum of $L=0$ states of two heavy and two light quarks

\begin{tabular}{|c|c|c|}
\hline$S_{h}$ & $S_{l}$ & $J^{P C}$ \\
\hline & & $2^{++}$ \\
1 & 1 & $1^{++}$ \\
& & $0^{++}$ \\
\hline 0 & 1 & $1^{+-}$ \\
\hline \hline 1 & 0 & $1^{+-}$ \\
\hline 0 & 0 & $0^{++}$ \\
\hline
\end{tabular}

can contribute $S_{h}=0$ or $S_{h}=1$ to the total spin of the system. In table 1, possible total spins for a four quark system with $L=0$ are shown. Due to the heavy quark symmetry, states that have the same heavy quark spin $S_{h}$ should have the same mass.

In Sect. 2, this degeneracy will be derived starting from a correlation function framework. The extension of this framework to study the isospin structure of $X(3872)$, and possible decays of $X(3872)$ that can be used to determine the its isospin structure will be discussed in Sect. 3. Sect. 4 is devoted to the conclusions.

\section{Degeneracies from the Correlation Functions}

To determine the mass of a meson in a field theoretical framework, a correlation function of the form

$$
\Pi(q)=i \int d^{4} x e^{i q x}\left\langle 0\left|\mathcal{T} j(x) j^{\dagger}(0)\right| 0\right\rangle
$$

is studied. In eq. $3, j(x)^{\dagger}$ is an operator, called an interpolating current, that can create the particle to be studied from the vacuum. By inserting a complete set of states into the correlation function, it can be written in the form

$$
\Pi(q)=\sum_{h} \frac{\langle 0|j| h(q)\rangle\left\langle h(q)\left|j^{\dagger}\right| 0\right\rangle}{q^{2}-m_{h}^{2}}
$$

where the sum is over all mesons that can be created from the vacuum by $j^{\dagger}$. As is seen from eq. 4 , the masses of the mesons correspond to poles in the correlation function. Hence, to show that two mesons are degenerate, it is enough to show that the poles that they generate in the correlation function overlap.

To study the correlation function, it is necessary to choose a suitable interpolating current. In the molecular picture, the $J^{P C}=1^{++}$state can be written as

$$
|X(3872)\rangle=\frac{1}{\sqrt{2}}\left(\left|D \bar{D}^{*}\right\rangle-\left|\bar{D} D^{*}\right\rangle\right)
$$

In terms of the quarks, this same state can also be written as

$$
|X(3872)\rangle=\left|\left(S_{c \bar{c}}=1 ; S_{q \bar{q}}=1\right) J=1\right\rangle
$$

i.e., the heavy quarks are coupled to total spin one, the light quarks are coupled to total spin one, and the two systems are coupled to give $J=1$. Following this description, a suitable operator for the $X(3872)$ meson can be chosen as:

$$
j_{\alpha \beta}^{q}=\bar{Q}^{a} \gamma_{\alpha} Q^{b} \bar{q}^{b} \gamma_{\beta} q^{a}
$$


where the color factors $a$ and $b$ are chosen to give colourless heavy-light mesons. This current can be decomposed into irreducible representations of the Lorentz group as $j_{\alpha \beta}^{q}=\sum_{n=0,1,2} j_{\alpha \beta}^{n}$ where $j_{\mu \nu}^{n}=\mathcal{P}_{\mu \nu ; \bar{\mu} \bar{\nu}}^{n}$ where

$$
\begin{aligned}
\mathcal{P}_{\mu v ; \bar{\mu} \bar{\nu}}^{2} & =\frac{1}{2}\left(g_{\mu \bar{\mu}} g_{\nu \bar{v}}+g_{\mu \bar{\nu}} g_{v \bar{\mu}}-\frac{1}{2} g_{\mu \nu} g_{\bar{\mu} \bar{\nu}}\right) \\
\mathcal{P}_{\mu v ; \bar{\mu} \bar{\nu}}^{1} & =\frac{1}{2}\left(g_{\mu \bar{\mu}} g_{\nu \bar{v}}-g_{\mu \bar{\nu}} g_{v \bar{\mu}}\right) \\
\mathcal{P}_{\mu v ; \bar{\mu} \bar{\nu}}^{0} & =\frac{1}{4} g_{\mu \nu} g_{\bar{\mu} \bar{\nu}}
\end{aligned}
$$

The current $j^{0}$ can couple to scalars, $j^{1}$ to spin- 1 particles and $j^{2}$ to spin- 0 , spin- 1 and spin- 2 particles. The correlation functions of the operators $j^{n}$ can be obtained by applying the projection operators $\mathcal{P}^{i}$ to the correlation function of the current given in eq. 7 .

Substituting eq. 7 into eq. 3, the correlation function becomes

$$
\begin{aligned}
\Pi_{\alpha \beta ; \gamma \delta} & =i \int d^{4} x e^{i q x}\left\langle 0\left|\mathcal{T} j_{\alpha \beta}(x) j_{\gamma \delta}^{\dagger}(0)\right| 0\right\rangle \\
& =i \int d^{4} x e^{i q x}\left\langle 0\left|\mathcal{T} \bar{Q}^{a}(x) \gamma_{\alpha} Q^{b}(x) \bar{q}^{b}(x) \gamma_{\beta} q^{a}(x) \bar{Q}^{d}(0) \gamma_{\gamma} Q^{e}(0) \bar{q}^{e}(0) \gamma_{\delta} q^{d}(0)\right| 0\right\rangle
\end{aligned}
$$

Defining $q=2 m_{Q} v+k$, where $v$ is the velocity of the meson, and taking the $m_{Q} \rightarrow \infty$ limit, the correlation function can be written as:

$$
\begin{array}{r}
\Pi_{\alpha \beta ; \gamma \delta}=i \int d^{4} x e^{i k x}\langle 0| \mathcal{T} \overline{h_{\bar{Q}}^{(-) a}}(x) \gamma_{\alpha} h_{Q}^{(+) b}(x) \bar{q}^{b}(x) \gamma_{\beta} q^{a}(x) \\
\overline{h_{Q}^{(+) d}}(0) \gamma_{\gamma} h_{\bar{Q}}^{(-) e}(0) \bar{q}^{e}(0) \gamma_{\delta} q^{d}(0)|0\rangle
\end{array}
$$

where $h_{Q}^{(+)}\left(h_{\bar{Q}}^{(-)}\right)$contain the annihilation (creation) operators of the $Q$ quark (anti-Q quark). As stated earlier, in the heavy quark limit, the spin degrees of freedom decouple from the theory. Hence, the $\gamma$ matrices corresponding to the heavy quark can be factored out of the correlation function:

$$
\begin{aligned}
\Pi_{\alpha \beta ; \gamma \delta} & =\operatorname{Tr}\left[\gamma_{\alpha} \frac{1+\not b}{2} \gamma_{\gamma} \frac{1-\not p}{2}\right]\left(\mathcal{R}_{1} g_{\beta \delta}+\mathcal{R}_{2} v_{\beta} v_{\delta}\right) \\
& =2\left(g_{\alpha \gamma}-v_{\alpha} v_{\gamma}\right)\left(\mathcal{R}_{1} g_{\beta \delta}+\mathcal{R}_{2} v_{\beta} v_{\delta}\right)
\end{aligned}
$$

where $\mathcal{R}_{i}$ are some unknown functions. It is crucial that in the heavy quark limit, the correlation function of the $j^{q}$ current can be written in terms of only two unknown functions.

To obtain the degeneracies, it is enough to obtain the correlation functions of the currents $j^{n}$ from eq. 13 by applying the projection operators defined in eqs. 8-10 and then identify the contributions of the mesons that can be created from the vacuum by applying the $j^{n}$ interpolating currents. The details 
can be found in [6]. The expression of $\mathcal{R}_{i}$ as a sum over their poles can be obtained as:

$$
\begin{aligned}
\mathcal{R}_{1} & =\frac{D^{2} / 2}{k v-\Lambda_{2^{++}}}=\frac{A^{\prime 2}}{k v-\Lambda_{1^{++}}} \\
\mathcal{R}_{1} & =-8 \frac{C^{2}}{k v-\Lambda_{0^{++}}}+\frac{2}{3} \frac{D^{2}}{k v-\Lambda_{2^{++}}} \\
\mathcal{R}_{1}-\mathcal{R}_{2} & =\frac{D^{2}}{k v-\Lambda_{2^{++}}}+\frac{2 F^{2}}{k v-\Lambda_{1^{-+}}}=\frac{2 A^{2}}{k v-\Lambda_{1^{-+}}}+\frac{2 A^{\prime 2}}{k v-\Lambda_{1^{++}}}
\end{aligned}
$$

where $\Lambda_{J^{P C}}$ is the binding energy of a meson with quantum numbers $J^{P C}$ and is defined as

$$
\Lambda_{J^{P C}}=\lim _{m_{Q} \rightarrow \infty}\left(m_{J^{P C}}-2 m_{Q}\right)
$$

The various expressions of the functions $\mathcal{R}_{i}$ are consistent with each other only if

$$
\Lambda_{2^{++}}=\Lambda_{0^{++}}=\Lambda_{1^{++}}
$$

The current in eq. 7 couples only to states with $C=+1$. To study any possible degeneracies with states that have $C=-1$, a possible current is

$$
\tilde{j}_{\alpha \beta}^{q}=\bar{Q}^{a} \gamma_{\alpha} \gamma_{5} Q^{b} \bar{q}^{b} \gamma_{\beta} q^{a}
$$

where a $\gamma_{5}$ is inserted between the heavy quark fields. In the heavy quark limit, the correlation function of this current can be written as:

$$
\tilde{\Pi}_{\alpha \beta ; \gamma \delta}=\operatorname{Tr}\left[\gamma_{\alpha} \gamma_{5} \frac{1+\not p}{2} \gamma_{\gamma} \gamma_{5} \frac{1-\not p}{2}\right]\left(\mathcal{R}_{1} g_{\beta \delta}+\mathcal{R}_{2} v_{\beta} v_{\delta}\right)
$$

where the functions $\mathcal{R}_{i}$ appearing in eq. 18 are the same functions that appear in eq. 13. This is because, the insertion of $\gamma_{5}$ only modifies the spin structure of the heavy quarks which is factored out of the correlation function. Carrying out the same procedure as before to this correlation functions, the previously obtained degeneracies are enlarged to $\Lambda_{0^{++}}=\Lambda_{1^{++}}=\Lambda_{2^{++}}=\Lambda_{1^{+-}}$and also $\Lambda_{0^{--}}=\Lambda_{1^{-+}}$. Which completes the proof that $X(3872)$ (the $J^{P C}=1^{++}$) state should have three degenerate partners.

Before concluding this section, it should be noted that the previous discussion is not limited to $X(3872)$; it is valid for any state that has two heavy quarks. Also it should be stated that, the previous discussion is not enough to prove (or disprove) the existence of $X(3872)$ (or any other resonance), but it states that if one such resonance exists, in the heavy quark limit, three other resonance should exist.

\section{Isospin Structure of $X(3872)$}

The decay pattern of $X(3872)$ suggests that it might have a sizeable $I=1$ component along with an $I=0$ component. Let $|X(1)\rangle$ and $|X(0)\rangle$ denote the $I=1$ and $I=0$ component of $X(3872)$. Then the wave function of $X(3872)$ and its orthogonal state can be written as:

$$
\begin{aligned}
|X(3872)\rangle & =\cos \theta|X(0)\rangle+\sin \theta|X(1)\rangle \\
\left|X_{\perp}\right\rangle & =-\sin \theta|X(0)\rangle+\cos \theta|X(1)\rangle
\end{aligned}
$$


where $\theta$ is the mixing angle. In [7], a method is proposed to calculate this mixing angle Let the interpolating currents for the $I=0$ and $I=1$ states be $j^{I}=1 / \sqrt{2}\left[j-(-1) j^{d}\right]$. Then the interpolating currents for the states in eqs. 19 and 20 would be:

$$
\begin{aligned}
j_{X(3872)} & =\cos \theta j^{I=0}+\sin \theta j^{I=1} \\
j_{X_{\perp}} & =-\sin \theta j^{I=0}+\cos \theta j^{I=1}
\end{aligned}
$$

Since these two physical states should be orthogonal, i.e. one should not evolve into the other one, the mixing angle $\theta$ can be determined from

$$
\begin{aligned}
& i \int d^{4} x e^{i q x}\left\langle 0\left|\mathcal{T} j_{X(3872)}(x) j_{X_{\perp}}^{\dagger}(0)\right| 0\right\rangle \\
& +i \int d^{4} x e^{i q x}\left\langle 0\left|\mathcal{T} j_{X_{\perp}}(x) j_{X(3872)}^{\dagger}(0)\right| 0\right\rangle=0
\end{aligned}
$$

Solving this equation for the mixing angle, it can be obtained as:

$$
\tan 2 \theta=\frac{\Pi^{10}+\Pi^{01}}{\Pi^{00}-\Pi^{11}}=\frac{\Pi^{u u}-\Pi^{d d}}{\Pi^{u d}+\Pi^{d u}}
$$

where $\Pi^{i j}$ denotes the correlation function

$$
\Pi^{i j}=i \int d^{4} x e^{i q x}\left\langle 0\left|\mathcal{T}_{j}^{i}(x) j^{j \dagger}(0)\right| 0\right\rangle
$$

In eq. 23 , it is understood that only the contribution of the $X(3872)$ is separated in the correlation function.

Explicit calculation of the correlation functions is beyond the scope of this work, but a qualitative estimate can be obtained. $\Pi^{u u}-\Pi^{d d}$ receives contributions only from isospin violating effects. Hence it has to be proportional to either the electromagnetic fine structure constant, $\alpha$, or to the quark mass difference $m_{u}-m_{d}$. Considering that the correlation function has mass dimension ten, the leading terms in $\Pi^{u u}-\Pi^{d d}$ should be either $\alpha \Lambda^{10}$ or $\left(m_{u}-m_{d}\right) \Lambda^{9}$ where $\Lambda$ is the Borel parameter in the heavy quark limit. Using a value $\Lambda=m_{X(3872)}-2 m_{c}$, both of these contribution are of the same order of magnitude. Hence, one can assume $\Pi^{u u}-\Pi^{d d} \simeq\left(m_{u}-m_{d}\right) \Lambda^{9}$. There is no such symmetry to constrain $\Pi^{u d}+\Pi^{d u}=\Pi^{00}-\Pi^{11}$. But note that $\Pi^{00}-\Pi^{11}$ is a measure of the difference between the $I=0$ and $I=1$ multiplet. Hence, it is safe to assume that $\Pi^{u d}-\Pi^{d u} \simeq \beta \Lambda^{10}$ where $\beta$ is a measure of the splitting between the $I=0$ and $I=1$ multiplets. Using these estimates in the eq. 23 , the mixing angle is estimated as

$$
\tan 2 \theta=\frac{m_{u}-m_{d}}{\beta \Lambda}
$$

Considering that the light quarks in the $X(3872)$ meson are in a vector configuration, an estimate of $\beta$ can be taken from the $\rho-\omega$ sistem. Defining $\beta$ as

$$
\beta \sim \frac{m_{\omega}-m_{\rho}}{m_{\omega}+m_{\rho}}=0.0046,
$$

the mixing angle would be estimated as:

$$
\tan 2 \theta \simeq 0.56 \Longrightarrow \theta \simeq 15^{\circ}
$$




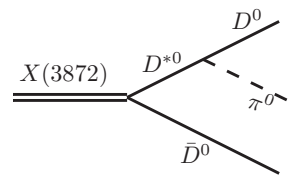

(a)

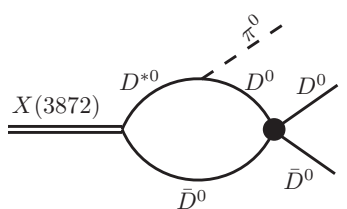

(b)

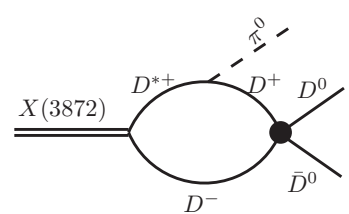

(c)

Figure 1. Diagrams contributing to the decay $X(3872) \rightarrow D^{0} \bar{D}^{0} \pi^{0}$ : (a) Tree level diagram, (b) and (c): final state interactions.

The mixing angle can also be calculated in the model of [8], where the wave function for the $X(3872)$ is defined as

$$
\begin{aligned}
|X(3872)\rangle & =\left|\psi_{1}\right\rangle\left|D^{0} \bar{D}^{* 0}\right\rangle+\left|\psi_{2}\right\rangle\left|D^{+} D^{*-}\right\rangle \\
& \left.=\frac{1}{\sqrt{2}}\left(\left|\psi_{1}\right\rangle+\left|\psi_{2}\right\rangle\right)|I=0\rangle+\frac{1}{\sqrt{2}}\left(\left|\psi_{1}\right\rangle-\left|\psi_{2}\right\rangle\right)\right)|I=1\rangle
\end{aligned}
$$

where the states $\left|\psi_{i}\right\rangle$ are normalised as

$$
\left\langle\psi_{1} \mid \psi_{1}\right\rangle+\left\langle\psi_{2} \mid \psi_{2}\right\rangle=1
$$

In terms of the wave functions $\psi_{1}$ and $\psi_{2}$, the mixing angle can be written as

$$
\tan ^{2} \theta=\frac{\int d^{3} r\left|\psi_{1}(\vec{r})-\psi_{2}(\vec{r})\right|^{2}}{\int d^{3} r\left|\psi_{1}(\vec{r})+\psi_{2}(\vec{r})\right|^{2}} \Rightarrow \theta \sim 39^{\circ}
$$

As can be seen in these two arguments, the isospin mixing in the $X(3872)$ state can be as large as maximal mixing $\theta_{\max }=45^{\circ}$. In the case of maximal mixing, one would naively expect that the ratio of the amplitudes in eq. 2 to be close to one. The suppression of this ratio is due to the fact that the decay amplitude into a final state that involves a $J / \psi$ is proportional to the wave function at the origin. Hence the decay of the $I=1$ component is proportional to $\psi_{1}(0)-\psi_{2}(0)$ which is suppressed relative to decay of the $I=0$ component.

In order to probe the isospin one component of the $X(3872)$, it is necessary to study a decay for which the amplitude is not proportional to the wave function at the origin. Such a decay is the decay $X(3872) \rightarrow D^{0} \bar{D}^{0} \pi^{0}$ which is the only kinematically allowed strong decay of $X(3872)$ in which the final state contains both of the $c$ quarks of the $X(3872)$ meson.

In [9] this decay is analysed in the model of [5] including both the tree level diagram and the final state interactions (see figure 1). The model, which is an heavy quark effective field theory, contains four low energy effective constants. Three of these constants can be fixed from $m_{X(3872)}$, the ratio in eq. 2 , and $m_{Z_{b}(10610)}$. Fixing the fourth parameter, $C_{0 A}$, would fix all the unknowns in this model.

In figures 2 and 3 , the dependence of the width $\Gamma\left(X(3872) \rightarrow D^{0} \bar{D}^{0} \pi^{0}\right)$ on $C_{0 A}$ is shown. The cutoff $\Lambda$ is used to regularise the contact interactions. The horizontal band is the width when final state interactions are ignored, and the other band is when they are included. The values of $C_{0 A}$ for which there are peaks correspond to values of $C_{0 A}$ where there exists a resonance in the $D^{0} \bar{D}^{0}$ scattering.

As can be seen from the figures, close to the resonance in the $D^{0} \bar{D}^{0}$ channel, the width of the $X(3872) \rightarrow D^{0} \bar{D}^{0} \pi^{0}$ decay is very sensitive to the value of this fourth parameter. Hence if a measurement of the width of the decay $X(3872) \rightarrow D^{0} \bar{D}^{0} \pi^{0}$ reveals a value for the width that deviates 


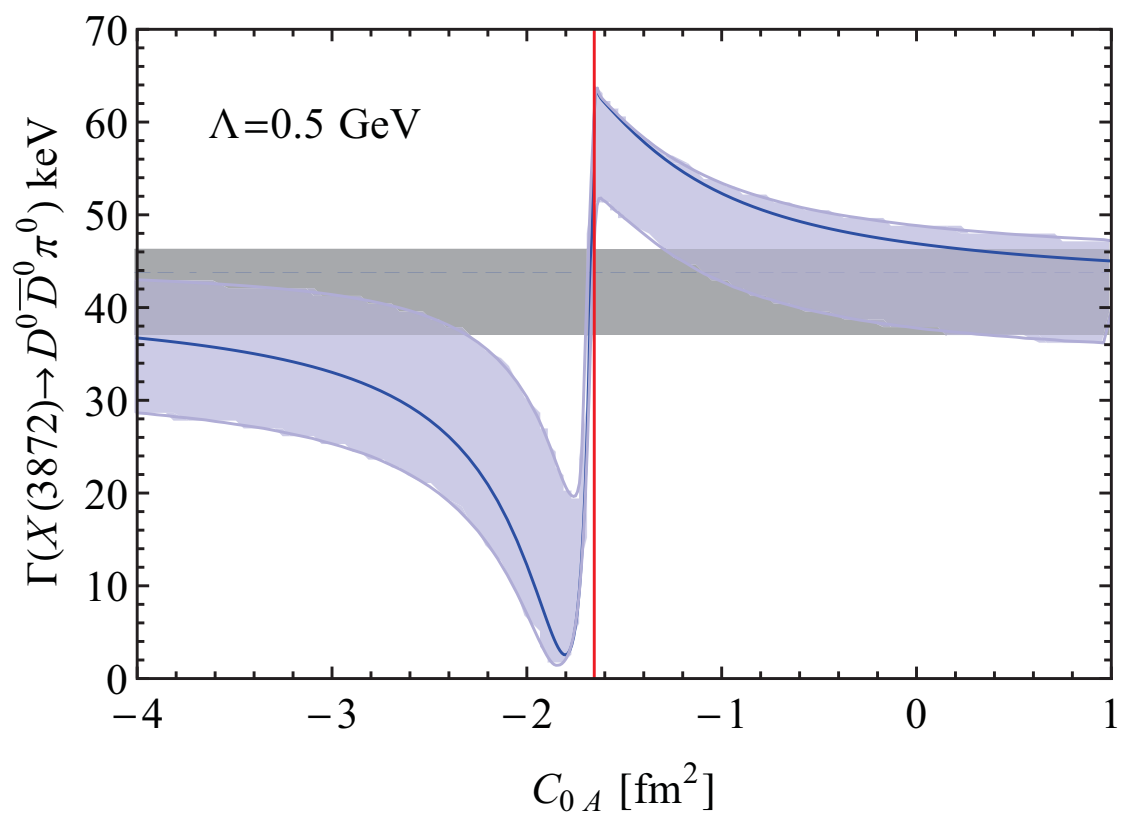

Figure 2. Dependence of the width of the $X(3872) \rightarrow D^{0} \bar{D}^{0} \pi^{0}$ decay on $C_{0 A}$ for the cutoff $\Lambda=0.5 \mathrm{GeV}$

significantly from the tree level value, the parameter $C_{0 A}$ can be fixed quite precisely allowing the determination of the isospin mixing angle in the $X(3872)$.

\section{Conclusion}

In this presentation, an analysis of the degenerate partners of $X(3872)$ is presented in the heavy quark limit within a framework that uses only quark-gluon degrees of freedom. Then this framework is extended to qualitatively analyse the isospin structure of $X(3872)$ and it is shown that the mixing angle can be very large. Finally, the $X(3872) \rightarrow D^{0} \bar{D}^{0} \pi^{0}$ decay is proposed to determine the isospin structure of the $X(3872)$ meson.

\section{References}

[1] S.-K. Choi et al. (Belle Collaboration), Phys. Rev. Lett. 91, 262001 (2003)

[2] R. Aaij et al. [LHCb Collaboration], Phys. Rev. Lett. 110 (2013) 222001.

[3] N. A. Tornqvist, Phys.Lett. B590, 209 (2004)

[4] C. Hidalgo-Duque, J. Nieves, E. Oset and R. Ruiz Arriola, Phys. Rev. D87 076006 (2013)

[5] J. Nieves and M. P. Valderrama, Phys. Rev. D86, 056004 (2012)

[6] C. Hidalgo-Duque, J. Nieves, A. Ozpineci and V. Zamiralov, "X(3872) and its Partners in the Heavy Quark Limit of QCD,” Phys. Lett. B 727, 432 (2013) [arXiv:1305.4487 [hep-ph]].

[7] T. M. Aliev, A. Ozpineci, and V. Zamiralov, Phys. Rev. D83, 016008 (2011)

[8] D. Gamermann, J. Nieves, E. Oset, and E. Ruiz Arriola, Phys. Rev. D81, 014029 (2010) 
EPJ Web of Conferences

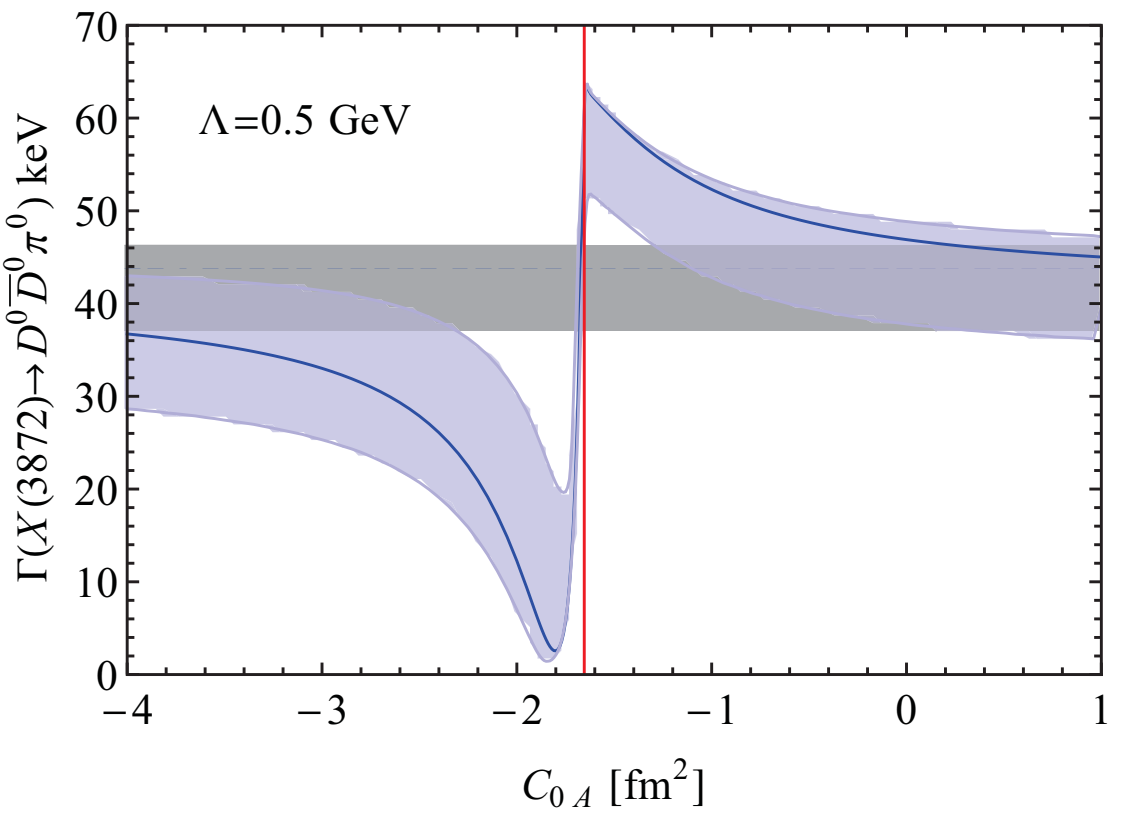

Figure 3. Same as figure 2 but for $\Lambda=1 \mathrm{GeV}$

[9] F.-K. Guo, C. Hidalgo-Duqeu, J. Nieves, A.Ozpineci, M.P. Valderrama, Eur. Phys. J. C74, 2885 (2014) 